\title{
Autosomal recessive peripheral sensory neuropathy in 3 non-Ashkenazi Jewish families*
}

\author{
ISRAEL TAMARI, RICHARD M GOODMAN, IDA SAROVA, \\ MARJORIE HERTZ, RAPHAEL ADAR, AND TIBOR ZVIBACH $\dagger$ \\ From the Departments of Medical Genetics, Neurology, Radiology, and Surgery, Chaim Sheba Medical \\ Center and the Sackler School of Medicine, Tel-Aviv University, Tel-Hashomer, Israel
}

SUMMARY Three unrelated Oriental Jewish families with a total of eight subjects with progressive hereditary sensory neuropathy are reported. The parents were all unaffected and because of parental consanguinity in each of the three families it is postulated that this rare neurological disorder is transmitted in an autosomal recessive manner. In one family both parents showed an abnormal response to pain stimulation with normal motor and sensory nerve conduction velocity. This response $\underset{\perp}{\mathcal{N}}$ may be an expression of the carrier state for this hereditary disease. Only five other families (non- 은 Jewish) have been reported as having this form of peripheral hereditary sensory neuropathy. These observations suggest that one type, the progressive form, of peripheral hereditary sensory neuropathy may be more common in Oriental Jews.

Several genetic diseases are known to have a high frequency among the various non-Ashkenazi Jewish communities. There is preliminary evidence to suggest that an autosomal recessive form of peripheral hereditary sensory neuropathy (PHSN) may be a new addition to this group of hereditary diseases common to Oriental Jews. Recently we have studied three unrelated Oriental Jewish families (two from Syria and one from Iraq) with a total of eight subjects with PHSN. Only five other such families have been reported as having the same form of PHSN. The purpose of this report is (1) to define clinically the features of this rare neurological disorder; (2) to present evidence for autosomal recessive transmission; and (3) to clarify and emphasise the genetic heterogeneity present within the broad grouping of PHSN. Additional comments will be made on possible detection of the heterozygous state based on electrophysiological studies showing an abnormal response to pain stimulation in unaffected consanguineous parents. The severe deformities of the limbs that result from this form of PHSN makes early diagnosis of crucial importance with respect to treatment.

\footnotetext{
*Supported in part by grants from the Lake Chemical Corp, Chicago, and The National Foundation for Jewish Genetic Diseases, New York City.

$\dagger$ Deceased.
}

Received for publication 23 January 1980
In 1883 Morvan $^{1}$ first described a patient who had $\vec{\bullet}$ a form of peripheral sensory neuropathy. Over tiee years various forms of peripheral sensory neuropath have been called the Morvan syndrome. On revie ing published reports it is apparent that there are several causes of peripheral sensory neuropathy, and that the all inclusive diagnosis of Morvan syndrome $\frac{\circ}{\varnothing}$

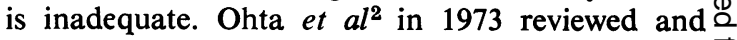
classified the various types of hereditary sensory $\overrightarrow{\overrightarrow{0}}$ neuropathy (HSN). They classified them into four 3 types: type I, autosomal dominant; type II, autosomal recessive; type III, familial dysautonomia; and type IV, congenital insensitivity to pain. In 1975, Thomas $^{3}$ in a review of HSN added a fifth typeco associated with the hereditary ataxias. Recently we $\frac{0}{3}$ have studied eight affected subjects from three nonrelated Jewish families with what appeared to be HSN type II. However, in attempting to apply Ohta's classification to our cases we found certain discrep-음 ancies. Our findings together with other such reported $>$ cases suggest that type II, or more specifically the recessive peripheral hereditary sensory neuropathy (PHSN) form, is composed of a non-progressive and a progressive form.

The purpose of this report is to give a detailed N clinical account of those members affected in our three families and to compare their findings witho similar patients previously described. Furthermore, $\mathbb{\mathbb { D }}$ efforts will be made to clarify and emphasise the? heterogeneity of PHSN. 




FIG 1 Family 1. (a) The pedigree,

$(b, c)$ amputation of some of the digits with flexion contractures of others in the proband (IV.6), (d) x-ray of the proband's hands showing bone lysis and absence of phalanges in some digits.

\section{Family studies}

\section{FAMILY 1}

All members except the proband of this Oriental Jewish family live in Syria at present. As can be seen in fig 1a the parents are consanguineous through an uncle-niece marriage. The proband has an older brother (IV.2) living in Syria who also has the same disorder.

\section{Case IV.2}

Information on this 36-year-old male is available only through the proband. She relates that when her affected brother was approximately 5 years old their parents noted that he had painless ulcers on his toes. Many painless episodes of trauma including burns of the digits produced suppurative ulcers with osteomyelitis resulting in the gradual loss of parts of the distal phalanges of the feet and hands. This patient is confined to a wheel chair because of bilateral amputation of the legs below the knees. In addition, both hands have been amputated above the wrists.

\section{Case IV.6}

The proband is an intelligent 23-year-old woman who was first seen in our peripheral vascular clinic 2 years ago. Since this time she has been evaluated extensively and all her physical findings are limited to the peripheral extremities. From the age of 4 years she has had problems related to painless ulcers of the hands and feet. Physical examination of her hands and feet show complete and partial amputation of some digits with deformity and flexion contractures of the others (fig 1b, c). Neurological examination showed impairment of the sensory modalities of touch, pain, and temperature involving the distal portions of her upper and lower extremities. Vibration and position were normal. Deep tendon reflexes were completely absent in the upper and lower limbs. The remainder of the neurological examination and all other aspects of the physical examination were normal.

$X$-ray studies of her hands and feet showed varying degrees of bone lysis with partial and complete absence of some portions of the digits and deformity of the others (fig 1d, table 1).

Electrophysiological studies showed normal motor nerve conduction velocity (NCV), normal distal latency, and severe impairment of sensory $\mathrm{NCV}$ (table 2).

FAMILY 2

This Oriental Jewish family originated from Syria (not related to family 1) and lives in Israel. The

TABLE $1 X$-ray findings in the extremities in the three affected families

\begin{tabular}{clllll}
\hline & Bone lysis & Absence of phalanx & $\begin{array}{l}\text { Absence of } \\
\text { digit }\end{array}$ \\
\hline $\begin{array}{c}\text { Family 1 } \\
\text { IV.6 }\end{array}$ & RH:2 & & RH:2,3,4 & LH:2 & RF:1 \\
Family 2 & RF:2,4 & LF:2 & RF:5 & LF:2,3 & \\
IV.2 & RH:1,2,3 & LH:1,2,3 & & & RF:1 \\
IV.4 & RF:1 & & LF:5 & & \\
$\begin{array}{c}\text { Family } 3 \\
\text { V.5 }\end{array}$ & LF:1 & & RF:1 & LF:2 & \\
V.6 & RF:1 & LF:1 & LF:1 & & \\
\hline
\end{tabular}

RH, right hand; LH, left hand; RF, right foot; LF, left foot. Numbers 1 to 5 refer to the exact digit involved. 
TABLE 2 Nerve conduction velocity studies in the three affected families

\begin{tabular}{|c|c|c|c|c|}
\hline & \multicolumn{2}{|l|}{ Motor } & \multicolumn{2}{|c|}{ Sensory } \\
\hline & Ulnar & Peroneal & Ulnar & Peroneal \\
\hline \multicolumn{5}{|l|}{ Family 1} \\
\hline IV. 6 & $72 *$ & 65 & $32 \cdot 5$ & 22 \\
\hline \multicolumn{5}{|l|}{ Family 2} \\
\hline IV. 2 & 61 & 52 & 42 & 33 \\
\hline IV.4 & 67 & 48 & 25 & 28 \\
\hline IV.6 & 75 & 70 & 70 & 64 \\
\hline \multicolumn{5}{|l|}{ Family 3} \\
\hline V.5 & 70 & 38 & 41 & 35 \\
\hline V.6 & 55 & 40 & 35 & 20 \\
\hline \multicolumn{5}{|l|}{ Normal } \\
\hline values & $60-70$ & $<45$ & $60-70$ & $<45$ \\
\hline
\end{tabular}

*The nerve velocity is in $\mathrm{m} / \mathrm{sec}$.

parents are first cousins (fig 2a). The mother has diabetes mellitus and the father died in a road accident. Four of the five children in this family have PHSN. None of these five offspring has diabetes mellitus.

All four affected members first noted painless ulcerations on their feet between the ages of 11 and 13 years. Because of many episodes of trauma they developed suppurative osteomyelitis resulting in various degrees of hand and foot deformities.

Case IV.2

The proband is a 22-year-old male who was first examined at our clinic at the age of 20 . At this time he had painless ulcers on his right hand involving the 1st, 2nd, and 3rd fingers and absence of his right big toe (fig $2 b, c)$.

\section{Case IV.4}

This 18-year-old female had painless ulcerations on the ventral surface of her right and left big toes and on the left 3rd and 5th toes.

Case IV.5

The twin sister of IV.4 also had a painless ulcer on the ventral surface of her right big toe and a healed ulcer on her left heel.

\section{Case IV.6}

This 11-year-old sister had a mild swelling at the base of her right big toe with mild impairment of sensation.

Neurological examination of these four affected sibs revealed impairment of pain, temperature, and touch sensation adjacent to the areas involved. All affected members of this family showed an absence of some deep tendon reflexes.

$X$-ray studies showed varying degrees of bone lysis with partial and complete absence of some digits (fig $2 \mathrm{~d}$, table 1 ).

Electrophysiological studies showed normal motoro $\mathrm{NCV}$ with impairment of sensory NCV in all the

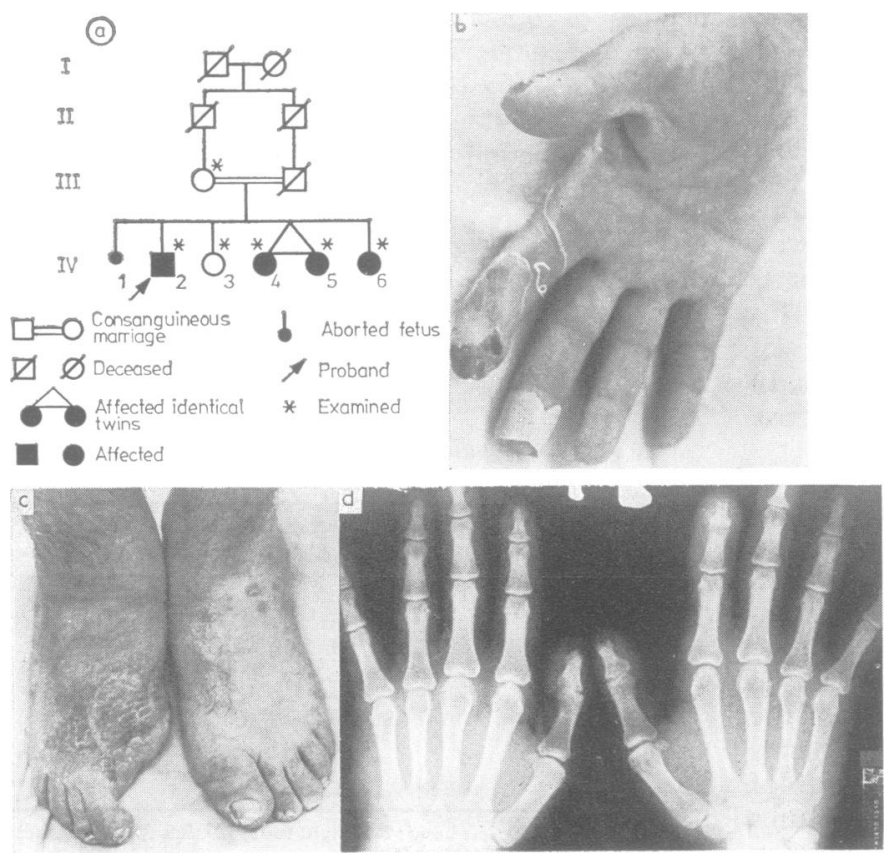

FIG 2 Family 2. (a) The pedigree, (b) ulceration of the distal portions of the 2nd and 3rd digits of the right hand of the proband (IV.2), (c) oedema of the right foot with amputated right big toe (IV.2), (d) $x$-ray of the proband's hands showing bone lysis in some digits. 

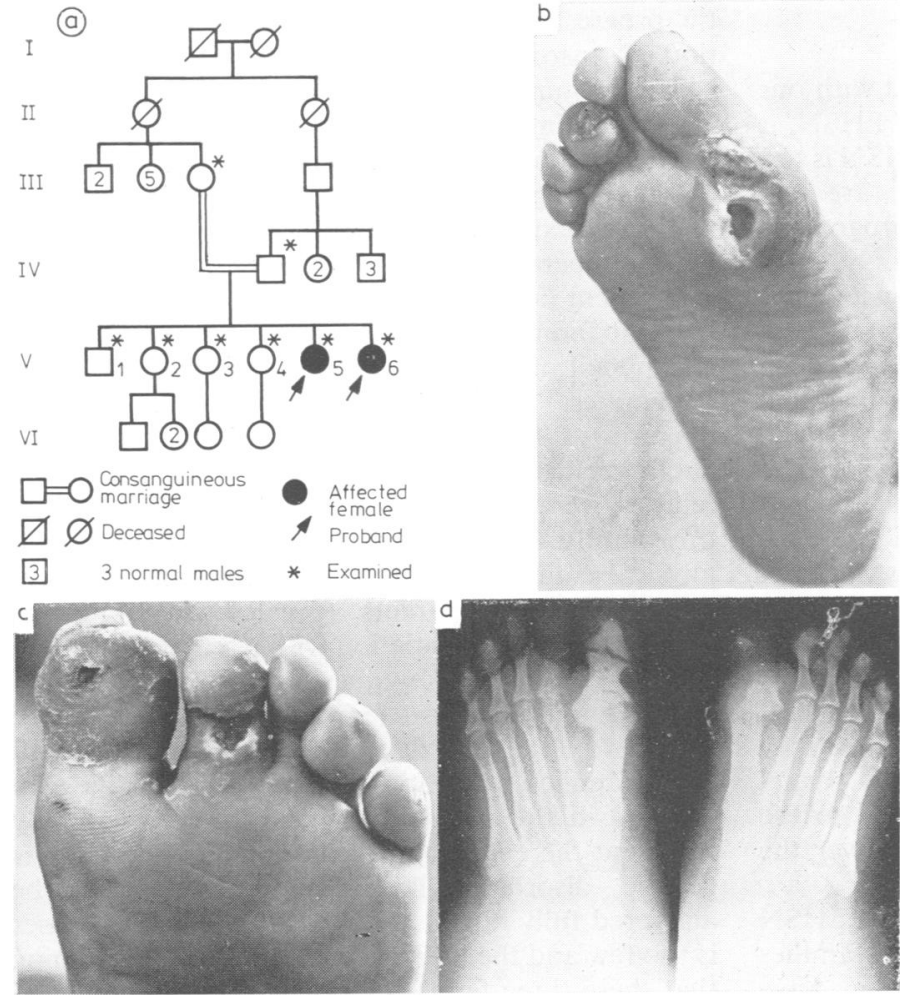



FIG 3 Family 3. (a) The pedigree, (b) ulceration about right sole and 3rd digit of foot in V.5, (c) partial absence and ulceration of left big toe and 2 nd digit $(V .5)$ (d) $x$-ray of the feet (V.6) showing bone lysis and absence of phalanges in some digits. affected members except IV.6, who had a normal sensory NCV despite the clinical findings of a mild sensory deficit (table 2).

\section{FAMILY 3}

This Oriental Jewish family originated from Iraq and lives in Israel. The parents are healthy and consanguineous (fig 3a). The two affected sibs V.5 and V.6 were first examined in our clinic at the ages of 19 and 16 years, respectively. They appeared to have normal intelligence and stated that from the age of 12 years they had noticed painless ulcerations on the soles of their feet. Multiple episodes of trauma to their feet have resulted in suppurative ulcers and osteomyelitis.

\section{Case V.5}

At the age of 19 this woman had a large ulcer on her right sole with partial absence of her left big toe and the distal portion of the 2 nd left toe (fig $3 b, c)$.

\section{Case V.6}

At the age of 21 years this woman had a deep ulcer on her left sole with absence of the distal phalanx of her left big toe.

Neurological examination of both sibs showed impairment of touch, pain, and temperature sensation bilaterally on the distal portions of the lowel extremities. Sib V.5 also had impairment of vibration and position sensation. The patellar reflexes were hyperactive in both sibs, while the other deep tendon reflexes were normal.

$X$-ray studies of their feet showed varying degrees of bone lysis with partial and complete absence of some digits (fig $3 \mathrm{~d}$, table 1 ). The upper extremities were normal.

Electrophysiological studies showed a mild decrease in motor NCV but severely impaired sensory NCV. There was no pain response to maximal current stimulus (table 2).

The parents and other unaffected sibs of family 3 had no neurological impairment. On electrophysiological testing both parents showed normal motor and sensory $\mathrm{NCV}$, but had no pain response to maximal electrical stimulation.

Autonomic nervous system function as expressed by heart rate, systemic blood pressure, and sweating was normal in all eight affected members from the three families. Electrocardiographic examinations and various biochemical determinations were also within normal limits. 


\section{Discussion}

After reviewing published reports, coupled with our own experience with the recessive form of PHSN, it seems that Ohta's classification of type II HSN is too generalised. In the recessive HSN there are two distinct subgroups, a congenital or non-progressive form and a late onset or progressive form.

In the non-progressive form six families with eight affected subjects have been described. ${ }^{4-8}$ Of these eight cases, Haddow and co-workers ${ }^{7}$ reported an affected brother and sister born to normal unrelated parents, and Murray ${ }^{8}$ gave an account of two affected sisters born to normal first cousin parents. The remaining four patients reported by Linarelli and Prichard, ${ }^{4}$ Barry et $a l,{ }^{5}$ and Person et al ${ }^{6}$ were all isolated cases in families where the parents were not related. Although the data are sparse, it would seem that this form of HSN falls within the confines of autosomal recessive inheritance.

Clinically, the non-progressive form has its onset of sensory impairment at birth. The sensory loss may involve all modalities ${ }^{48}$ or may show only partial involvement. The disorder may be confined to the limbs alone ${ }^{6}$ or show diffuse involvement over the entire body. 4578

The second subgroup of the recessive type of HSN is the progressive form. Our three affected families have this form and five other such families have been reported. ${ }^{29-12}$ Thus there is a total of eight families with 18 affected subjects with the progressive form. Table 3 summarises the genetic and clinical findings in these eight affected families.

In contrast to the non-progressive form which has its onset at birth, the progressive form begins during childhood. As in the non-progressive form, the progressive type may vary in its bodily distribution of sensory impairment (see table 3).

The two unrelated Jewish families (families 1 and 2) originating from Syria have identical clinical manifestations of the disease consisting of sensory loss involving the distal portions of both the upper and lower extremities. In addition to impairment of pain, temperature, and touch sensation there were also absent deep tendon reflexes.

In the family from Iraq (family 3 ) the sensory loss is confined only to the distal portions of the lower extremities. One affected sib also had sensory impairment in vibration and position. Both sibs had hyperactive patellar reflexes while the other reflexes were normal. The unaffected members of this family showed no neurological findings on physical examination. However, electrophysiological studies in both parents revealed an abnormal response to pain stimulation with normal motor and sensory NCV. Two key questions arise with regard to the findings in this family as compared to the other two. (1) Is the disease process the same in all three families? (2) Could the electrophysiological findings in the parents of the Iraqi Jewish family represent the expression of the carrier state for this genetic disorder? These questions cannot be answered fully at this time for the number of cases is too few and the information too sparse. Assuming that these three families have the same genetic disorder, then presence of close parental consanguinity with presumably normal carrier parents would support an autosomal recessive mode of inheritance for this type of HSN.

The fact that three of the eight families described with the progressive form of recessive HSN are of Oriental Jewish background raises the possibility

TABLE 3 Reported cases with late onset of autosomal recessive peripheral sensory neuropathy

\begin{tabular}{|c|c|c|c|c|c|c|c|}
\hline & \multirow{2}{*}{$\begin{array}{l}\text { Johnson and } \\
\text { Spalding } 9\end{array}$} & \multirow{2}{*}{$\begin{array}{l}\text { Schoene } \\
\text { et al } 10\end{array}$} & \multirow{2}{*}{$\begin{array}{l}\text { Dyck } 11 \\
\text { Ohta et al2 }\end{array}$} & \multirow{2}{*}{$\begin{array}{l}\text { Jedrzejowska } \\
\text { and Milczarek }\end{array}$} & \multicolumn{3}{|l|}{ Present report } \\
\hline & & & & & Family 1 & Family 2 & Family 3 \\
\hline \multicolumn{8}{|l|}{ Postulated autosomal } \\
\hline recessive & + & + & + & + & + & + & + \\
\hline No of families & 2 & 1 & 1 & 1 & 1 & 1 & 1 \\
\hline Total No of cases & 2 & 2 & 4 & 2 & 2 & 4 & 2 \\
\hline Sex ratio F:M & $0: 2$ & $2: 0$ & $1: 3$ & $0: 2$ & $1: 1$ & $3: 1$ & $2: 0$ \\
\hline Age of onset (years) & $3-5$ & $11-13$ & $3-9$ & $2-4$ & $4-5$ & $11-13$ & 12 \\
\hline Progressive & + & + & + & + & + & + & + \\
\hline Distribution of sensory loss & $\begin{array}{l}\text { limbs and } \\
\text { trunk }\end{array}$ & limbs & $\begin{array}{l}\text { limbs and } \\
\text { trunk }\end{array}$ & distal limbs & distal limbs & distal limbs & $\begin{array}{l}\text { distal lower } \\
\text { limbs }\end{array}$ \\
\hline \multicolumn{8}{|l|}{ Modalities impaired } \\
\hline Pain and temperature & + & + & + & + & + & + & + \\
\hline Touch & + & + & + & + & + & + & + \\
\hline $\begin{array}{l}\text { Vibration and position } \\
\text { Tendon reflexes }\end{array}$ & $\underset{\text { absent }}{+}$ & $\begin{array}{l}+ \\
\text { absent }\end{array}$ & $\overline{\text { absent }}$ & hyperactive & $\overline{\text { absent }}$ & $\begin{array}{l}- \\
\text { partially } \\
\text { absentt }\end{array}$ & $\begin{array}{l}+,-^{*} \\
\text { patellar } \\
\text { hyperactive }\end{array}$ \\
\hline
\end{tabular}

* In one sister impaired, in the other one normal.

tThe other reflexes were normal 
that this rare genetic disease might be more common in Oriental Jews. It is known that a number of genetic diseases have a high frequency in certain ethnic segments of the Jewish population. ${ }^{13}$

Histological studies of affected sensory neurons in type II HSN have shown degeneration of myelinated fibres in cutaneous nerves with remyelination and patchy fibrous tissue surrounding the affected nerve. ${ }^{2} 1012$ The basic defect in all forms of HSN is not known, but Siggers and co-workers ${ }^{14}$ have shown a qualitative abnormality of one of the nerve growth factor (NGF) subunits, $\beta-\mathrm{NGF}$, in familial dysautonomia (type III HSN). It is too early to know if this abnormality underlies the basic defect in familial dysautonomia or other abnormalities, ${ }^{15}$ but if there is a factor which aids in promoting the differentiation and maturation of nerve tissue, perhaps there might be a factor necessary for maintaining function of nerve tissue. If such a factor were abnormal or decreased this might account for the sensory loss in some forms of HSN.

In conclusion, the severe deformities of the limbs that may result from the progressive type of HSN makes early diagnosis of crucial importance, with respect to informing such patients of the need to avoid all forms of trauma to their digits and to seek medical care when such incidents occur.

The authors are grateful to Mrs Ruth Grossman for her secretarial aid and to Mrs Hannah Levy for the photography.

\section{References}

1 Morvan A. De la analgesiaue a panaris des extrémités superieures ou pareso analgesie des extrémités superieures. Gaz Hebd Med 1883;20:580-3, 590-4, 624-6.
2 Ohta M, Ellefson RD, Lambert EH, Dyck PJ. Hereditary sensory neuropathy type II. Clinical, electrophysiologic, histologic and biochemical studies of a Quebec kinship. Arch Neurol 1973;29:23-7.

3 Thomas PK. Peripheral neuropathy. In: Matthews WB, ed. Recent advances in clinical neurology. Edinburgh: Churchill Livingstone, 1975:253-83.

4 Linarelli LG, Prichard JW. Congenital sensory neuropathy, complete absence of superficial sensation. Am J Dis Child 1970;119:513-20.

5 Barry JE, Hopkins IJ, Neal W. Congenital sensory neuropathy. Arch Dis Child 1973;49:128-32.

6 Person JR, Rogers RS, Rhodes KH. Congenital sensory neuropathy. Report of an atypical case. Arch Dermatol 1977;113:954-7.

7 Haddow JE, Shapiro SR, Gall DG. Congenital sensory neuropathy in siblings. Pediatrics $1970 ; 45: 651-5$.

8 Murray TJ. Congenital sensory neuropathy. Brain 1973;96:387-94.

9 Johnson RH, Spalding JMK. Progressive sensory neuropathy in children. J Neurol Neurosurg Psychiatry 1964; 27:125-30.

10 Schoene WC, Asbury AK, Astrom KE, Masters R. Hereditary sensory neuropathy. A clinical and ultrastructural study. $J$ Neurol Sci 1970;11:463-87.

11 Dyck PJ. Histologic measurements and fine structure of biopsied sural nerve: normal and in peroneal muscular atrophy, hypertrophied neuropathy and congenital sensory neuropathy. Mayo Clin Proc 1966;41:742-74.

12 Jedrzejowska $\mathbf{H}$, Milczarek $\mathbf{H}$. Recessive hereditary sensory neuropathy. J Neurol Sci 1976;29:371-87.

13 Goodman RM. Genetic disorders among the Jewish people. Baltimore: Johns Hopkins University Press, 1979.

14 Siggers DC, Rogers JG, Boyer SH, et al. Increased nerve growth factor $\beta$-chain cross-reacting material in familial dysautonomia. $N$ Engl J Med 1976;295:629-34.

15 Levi-Montalcini R. The nerve growth factor: its widening role and place in neurobiology. Adv Biochem Psychopharmacol 1976;15:237-51.

Requests for reprints to Professor Richard $M$ Goodman, Department of Medical Genetics, Sheba Medical Center, Tel-Hashomer, Israel. 